\title{
Quantitative study of the AMS-02 electron/positron spectra: implications for the pulsar and dark matter properties
}

\author{
Su-Jie Lin, Qiang Yuan, and Xiao-Jun Bi \\ Key Laboratory of Particle Astrophysics, Institute of High Energy Physics, \\ Chinese Academy of Science, Beijing 100049, P.R.China
}

(Dated: July 27, 2018)

\begin{abstract}
The AMS-02 has just published the unprecedentedly precise measurement of the cosmic electron and positron spectra. In this paper we try to give a quantitative study on the AMS-02 results by a global fitting to the electron and positron spectra, together with the updated positron fraction data. The Markov Chain Monte Carlo algorithm is adopted to do the fitting. The primary electron spectrum and the parameters for pulsars or dark matter which contribute extra positrons are determined simultaneously. We find that there is a hardening of the primary electron spectrum at $\sim 60$ $\mathrm{GeV}$. With such a new feature at the background spectrum both the pulsars and dark matter can explain the AMS-02 results very well. The dark matter scenario shows a drop at positron fraction at $\sim 300 \mathrm{GeV}$, however, suffers very strong constraints from Fermi $\gamma$-ray observations. The fitting results also suggest that the propagation model with convection may be more favored by the lepton data than the reacceleration model.
\end{abstract}

PACS numbers:

\section{INTRODUCTION}

There were large progresses in the measurements of the cosmic ray $(\mathrm{CR})$ lepton fluxes in recent years. Satellite experiments such as the Payload for Antimatter Matter Exploration and Light-nuclei Astrophysics (PAMELA) and the Fermi Large Area Telescope (Fermi-LAT), as well as the balloon-borne detector such as the Advanced Thin Ionization Calorimeter (ATIC) and the ground-based Cherenkov telescopes like the High Energy Stereoscopic System (HESS) and the Major Atmospheric Gamma-ray Imaging Cherenkov Telescopes (MAGIC), have improved the uncertainties of the measurements from order of magnitude down to several tens of percents [1-7]. The space station experiment Alpha Magnetic Spectrometer (AMS02), launched in May 2011, further improve the measurement precision of the CR fluxes by an order of magnitude due to larger exposure and much better control of the systematics [8]. With the AMS-02 result, we could perform the study of CRs in a more quantitative way instead of the qualitative studies [9-12].

The most interesting features found in the CR leptons are the excess of the positrons compared with the secondary background expectation from CR nuclei interaction with the interstellar medium (ISM) [1, 8, 13, 14]. Combining with the electron (or total electron/positron) spectra [2, 3, 5-7] implies that there should be extra sources emitting electron-positron pairs. The proposed models of the extra sources include the astrophysical sources such as pulsars [15-19], interaction occurring around the CR acceleration sources [20-22], as well as the dark matter (DM) annihilation/decay [23-28]. One can refer to the reviews for detailed description of the relevant models to explain the electron/positron excesses [29-33].

Given the data are more abundant and precise, we developed a global fitting tool which employs a Markov
Chain Monte Carlo (MCMC, [34]) method to sample the high-dimensional parameter space of the CR propagation and injection $[35,36]$. When applying in the study of the electron/positron excesses, such a global fitting method can fit both the background and the extra source parameters simultaneously and avoid the bias of choosing the background parameters. This approach definitely makes sense on the quantitative level, in spite that there are still uncertainties from various kinds of model configurations such as the CR propagation and the solar modulation [37]. It is expected that with better understandings of those issues based on more and better data from AMS02, the global fitting method may be more powerful to probe the underlying physical nature of the CRs.

One potential problem of the previous studies about the CR leptons is the systematical uncertainties among different detectors. As shown by the preliminary data of AMS-02 presented in 2013 International Cosmic Ray Conference [38], many kinds of measurements showed differences compared with previous measurements. Furthermore, the data-taking periods of various experiments are also different and the solar modulation effect will be different. It is no longer a problem after the most recent data release about the positron and electron fluxes by AMS-02 [39-41]. In this work we adopt the AMS-02 data about the positron fraction, positron plus electron flux, positron flux and the electron flux to study the injection properties of the backgrounds and the extra sources of the CR leptons.

This paper is organized as follows. We first give a description of our fitting process in Sec. II. The propagation of CRs in the Galaxy is introduced in section III. The assumptions and parameterization of the backgrounds and extra sources of electrons/positrons are described in section IV and section V. The fitting results in different models are given in section VI. We give some discussions about the results in section VII, and conclude in 
section VIII.

\section{THE SCHEME OF THE GLOBAL FITTING}

The scheme of the global fitting follows our previous study of the AMS-02 positron fraction results [9]. The model is described by a set of parameters $\vec{\theta}$, which include the primary electron spectrum, the electron/positron spectrum from the extra sources such as pulsar-like astrophysical sources or the DM. These parameters will be defined in the next sections. Once the parameters are given we can calculate the propagation of the CRs in the Milky Way. The production and propagation of secondary positrons/electrons will also be calculated at the same time. Then we compare the predicted spectra with the AMS-02 data and evaluate the model by minimizing the $\chi^{2}$.

The MCMC technique is used to derive the posterior probability distributions of the parameters from the observational data. According to the Bayes theorem, the posterior probability of a set of parameters $\vec{\theta}$ in light of the observational data is $\mathcal{P}(\vec{\theta} \mid D) \propto \mathcal{P}(D \mid \vec{\theta}) \mathcal{P}(\vec{\theta})$, where $\mathcal{P}(D \mid \vec{\theta})=\mathcal{L}(\vec{\theta}) \propto \exp \left(-\chi^{2}(\vec{\theta}) / 2\right)$ is the likelihood function of model $\vec{\theta}$ for the data, and $\mathcal{P}(\vec{\theta})$ is the prior probability of the model parameters before the current observations. In this work we adopt flat (constant) prior probabilities of all the model parameters in specified ranges (some of them are logarithmical, see details in the tables below).

We adopt the Metropolis-Hastings algorithm to generate the Markov chains from the unknown target distribution. The Metropolis-Hastings algorithm adopts a propose-and-accept process, in which the acceptance or rejection of a proposed point depends on the probability ratio between this point and the former one, to generate the chains. Such a sampling method can still work efficiently when the dimension of the parameter space is high.

The propagation model parameters will be first determined by fitting the $\mathrm{B} / \mathrm{C}$ and ${ }^{10} \mathrm{Be} /{ }^{9} \mathrm{Be}$ data (see more detailed description in section III). The propagation parameters are then fixed to be the best-fitting values when fitting the lepton data. The proton injection spectrum is also determined by fitting the AMS-02 data [38]. Therefore in the fitting process the parameter space $\vec{\theta}$ includes only the parameters of the lepton sector.

The global fitting gives us information of the background and properties of the extra sources at the same time. Therefore results on the astrophysical sources and DM are not biased due to the choice of background. The parameters for DM given in the work can be taken as the starting point for the future model-building. The results for pulsars can also be a guideline for pulsar model study although the case for pulsars is more complicated as each pulsar may have different properties. What we get may indicate the property of a nearby pulsar which gives dominant contribution to the positron excess.

\section{THE PROPAGATION OF COSMIC RAYS IN THE GALAXY}

Galactic CR particles diffuse in the Galaxy after being accelerated, suffering from the fragmentation and energy loss in the ISM and/or the interstellar radiation field (ISRF) and magnetic field, decay and possible reacceleration or convection. Denoting the density of CRs per unit momentum interval as $\psi$, the propagation can be described by the propagation equation

$$
\frac{\partial \psi}{\partial t}=Q(\mathbf{x}, p)+\nabla \cdot\left(D_{x x} \nabla \psi-\mathbf{V}_{c} \psi\right)+\frac{\partial}{\partial p} p^{2} D_{p p} \frac{\partial}{\partial p} \frac{1}{p^{2}} \psi-\frac{\partial}{\partial p}\left[\dot{p} \psi-\frac{p}{3}\left(\nabla \cdot \mathbf{V}_{c} \psi\right)\right]-\frac{\psi}{\tau_{f}}-\frac{\psi}{\tau_{r}}
$$

where $Q(\mathbf{x}, p)$ is the source distribution, $D_{x x}$ is the spatial diffusion coefficient, $\mathbf{V}_{c}$ is the convection velocity, $D_{p p}$ is diffusion coefficient in the momentum-space, $\tau_{f}$ and $\tau_{r}$ are the characteristic time scales used to describe the fragmentation and radioactive decay. The convection velocity $\mathbf{V}_{c}$ is generally assumed to linearly depend on the distance away from the Galaxy disk. The diffusion coefficient can be parameterized as $D_{x x}=D_{0} \beta\left(R / R_{0}\right)^{\delta}$, where $\beta$ is the velocity of the particle in unit of light speed $c, R \equiv p c / Z e$ is the rigidity. The reacceleration effect is described with the diffusion in momentum space. Considering the scenario in which the $\mathrm{CR}$ particles are reaccelerated by colliding with the interstellar random weak hydrodynamic waves, the relation between the spatial diffusion coefficient $D_{x x}$ and the momentum diffusion coefficient $D_{p p}$ can be expressed as [42, 43]:

$$
D_{p p} D_{x x}=\frac{4 p^{2} v_{A}^{2}}{3 \delta\left(4-\delta^{2}\right)(4-\delta) \omega},
$$

where $v_{A}$ is the Alfven velocity and the parameter $\omega$ is used to characterize the level of the interstellar turbulence. Since only $v_{A}^{2} / \omega$ is relevant, we adopt $\omega=1$ and refer $v_{A}$ to characterize the reacceleration. Free escape is assumed at the boundaries, $R_{h}$ and $z_{h}$, for cylindrical geometry.

The secondary-to-primary ratios of nuclei are almost independent of the injection spectrum. They are always 
TABLE I: The mean values and $1 \sigma$ uncertainties of the propagation parameters derived through fitting the $\mathrm{B} / \mathrm{C}$ and ${ }^{10} \mathrm{Be} /{ }^{9} \mathrm{Be}$ ratios. In the $\mathrm{DC}$ scenario $\delta$ is set to be 0 when $R$ is below $R_{0}$.

\begin{tabular}{llll}
\hline \hline & & $\mathrm{DR}$ & $\mathrm{DC}$ \\
\hline$D_{0}$ & $\left(10^{28} \mathrm{~cm}^{2} \mathrm{~s}^{-1}\right)$ & $6.58 \pm 1.27$ & $1.95 \pm 0.50$ \\
$\delta$ & & $0.333 \pm 0.011$ & $0.510 \pm 0.034$ \\
$R_{0}$ & $(\mathrm{GV})$ & 4 & $4.71 \pm 0.8$ \\
$v_{A}$ & $\left(\mathrm{~km} \mathrm{~s}^{-1}\right)$ & $37.8 \pm 2.7$ & - \\
$\mathrm{d} V / \mathrm{d} z$ & $\left(\mathrm{~km} \mathrm{~s}^{-1} \mathrm{kpc}^{-1}\right)$ & - & $4.2 \pm 3.2$ \\
$z_{h}$ & $(\mathrm{kpc})$ & $4.7 \pm 1.0$ & $2.5 \pm 0.7$ \\
$\phi$ & $(\mathrm{MV})$ & $326 \pm 36$ & $182 \pm 25$ \\
\hline \hline
\end{tabular}

employed to constrain the propagation parameters in Eq. (1). Generally used are the Boron-to-Carbon ratio (B/C) and unstable-to-stable Beryllium ratio $\left({ }^{10} \mathrm{Be} /{ }^{9} \mathrm{Be}\right)$. The $\mathrm{B} / \mathrm{C}$ ratio is sensitive to the average path of the CR particles go through between the source and the observer, which correlate positively with both $D_{x x}$ and the diffusion halo size $z_{h}$. The ${ }^{10} \mathrm{Be} /{ }^{9} \mathrm{Be}$ ratio is sensitive to probe the resident time of particles in the Galaxy, which correlate positively with $z_{h}$ but negatively with $D_{x x}$. Therefore, combining these two ratios, the main propagation properties can be fixed.

The major parameters to describe the propagation are $\left(D_{0}, \delta, v_{A}, \mathrm{~d} V / \mathrm{d} z, z_{h}\right)$. Since there are degeneracies between the models with reacceleration and convection effects, and the current data of $\mathrm{B} / \mathrm{C}$ and ${ }^{10} \mathrm{Be} /{ }^{9} \mathrm{Be}$ are not effective enough to distinguish them, we adopt two distinct scenarios as benchmark models of the propagation. They are referred as diffusion reacceleration (DR) model and diffusion convection (DC) model, respectively.

The public numerical tool, GALPROP version $54.1 .984^{1}[44,45]$, is adopted to calculate the propagation of $\mathrm{CR}$ particles. We employ the $\mathrm{B} / \mathrm{C}$ data from AMS-02 [38] and ACE [46], and the ${ }^{10} \mathrm{Be} /{ }^{9} \mathrm{Be}$ data from experiments ACE [47], Balloon [48-50], IMP7\&8 [51], ISEE3HKH [52], ISOMAX [53], Ulysses-HET [54] and Voyager [55] to constrain the propagation parameters. The MCMC method is adopted to fit the $\mathrm{B} / \mathrm{C}$ and ${ }^{10} \mathrm{Be} /{ }^{9} \mathrm{Be}$ data. In order to reproduce the low energy $\mathrm{B} / \mathrm{C}$ data, a broken power law, where $\delta$ is 0 when $R$ is below $R_{0}$, is adopted for $D_{x x}$ in the DC scenario [56]. To describe the propagation of $\mathrm{CR}$ particles in the solar system, we adopt the force-field approximation [57], which contains only one free parameter, the so-called solar modulation potential $\phi$. The mean values and $1 \sigma$ errors of the propagation parameters for DR and DC scenarios are shown in Table I. Fig. 1 shows the fitting results of B/C (left) and ${ }^{10} \mathrm{Be} /{ }^{9} \mathrm{Be}$ (right) ratios within $2 \sigma$ confidence level, compared with the observational data.

\footnotetext{
${ }^{1}$ Available at http://galprop.stanford.edu/
}

\section{THE PARAMETERS FOR THE BACKGROUND $e^{+}$AND $e^{-}$SPECTRA}

Electrons are also expected to be accelerated during the acceleration of $\mathrm{CR}$ nuclei at the sources, e.g. supernova remnants (SNRs). During the propagation, the inelastic collision between the nuclei and the ISM will produce secondary electrons and positrons. These components consist of the background contribution of electrons and positrons. Such a picture is supported by the observations of secondary-to-primary ratio of nuclei as well as the diffuse $\gamma$-ray emission [58, 59].

The spatial distribution of the injected CR particles is assumed to follow the SNR distribution

$$
f(r, z)=\left(\frac{r}{r_{\odot}}\right)^{a} \exp \left(-b \cdot \frac{r-r_{\odot}}{r_{\odot}}\right) \exp \left(-\frac{|z|}{z_{s}}\right),
$$

where $r_{\odot}=8.5 \mathrm{kpc}$ is the distance from the Sun to the Galactic center, $z_{s} \approx 0.2 \mathrm{kpc}$ is the characteristic height of Galactic disk. The two parameters $a$ and $b$ are chosen to be 1.25 and 3.56 following [60], which are adjusted to fit the $\gamma$-ray gradient. The injection spectra of all kinds of nuclei are assumed to be a broken power law form

$$
q_{\mathrm{i}}=N_{\mathrm{i}} \times\left\{\begin{array}{l}
\left(\frac{R}{R_{\mathrm{br}}^{\mathrm{p}}}\right)^{-\nu_{1}} \quad R \leq R_{\mathrm{br}}^{\mathrm{p}} \\
\left(\frac{R}{R_{\mathrm{br}}^{\mathrm{p}}}\right)^{-\nu_{2}} \quad R>R_{\mathrm{br}}^{\mathrm{p}}
\end{array}\right.
$$

where i denote species of the nuclei, $R$ is the rigidity of the particle, and $N_{\mathrm{i}}$ is the normalization constant proportional to the relative abundance of the corresponding nuclei. The injection is simply assumed to be stable, which is an effective approximation if the production rate of SNRs is high enough. Therefore, the injection source function can then be written as $Q_{\mathrm{i}}(\mathbf{x}, p)=f(r, z) q_{\mathrm{i}}(p)$.

Adopting the propagation parameters as the bestfitting values shown in Table I, we then constrain the injection parameters of Eq. (4) with the proton flux of AMS-02 [38]. The resulting nuclei injection parameters are given in Table II. Fig. 2 shows the best-fitting results and the $95 \%$ confidence ranges of the proton fluxes, compared with the AMS-02 measurement.

The secondary production of electrons and positrons can then be calculated with the propagated proton (and Helium) spectra. We use the parameterization presented in [61] to calculate the production spectrum of secondary electrons and positrons. To partially take into account the uncertainties when calculating the secondary fluxes, from e.g., proton-proton collision cross section, enhancement factor from heavier nuclei, and/or the propagation uncertainties, we employ a parameter $c_{e^{+}}$to re-scale the calculated secondary flux to fit the data. Note that the above mentioned uncertainties may not be simply represented with a constant factor, but most probably they are energy dependent $[62,63]$. Here a constant factor is just an approximation and the purpose is to fit the data. 

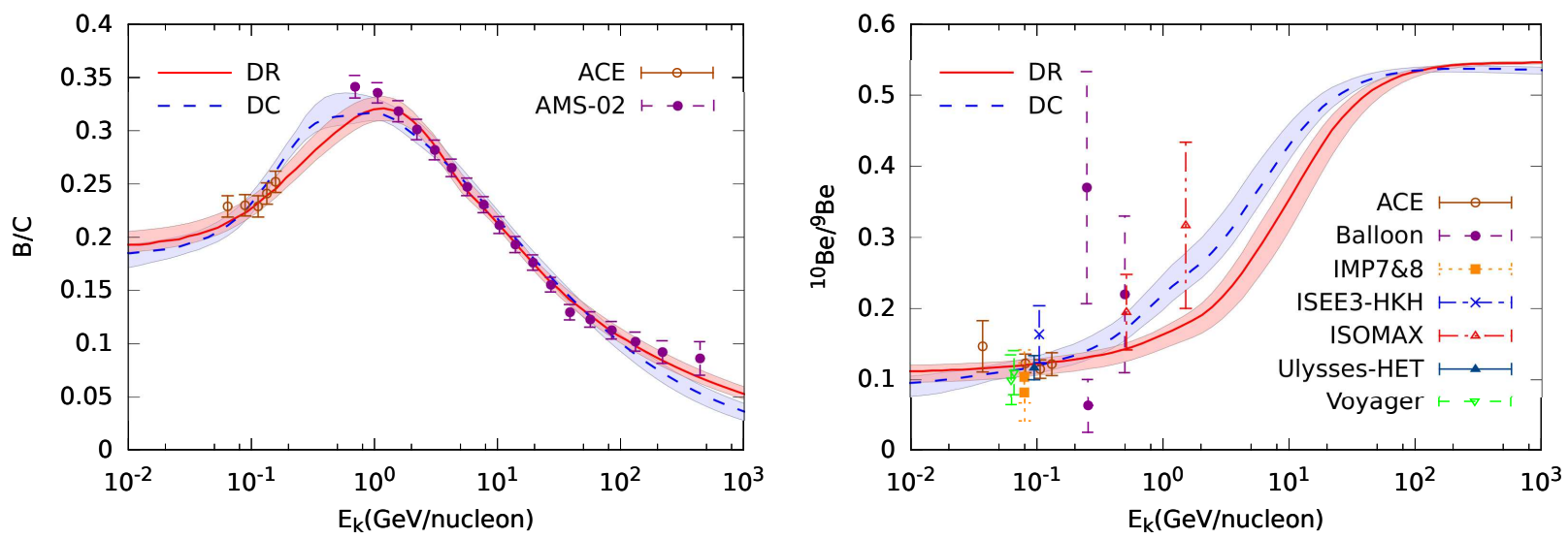

FIG. 1: The B/C ratio (left) and ${ }^{10} \mathrm{Be} /{ }^{9} \mathrm{Be}$ ratio (right) for the corresponding parameters shown in Table I, compared with the data. The bands show the $95 \%$ confidence ranges. The B/C data are from AMS-02 [38] and ACE [46], and the ${ }^{10} \mathrm{Be} /{ }^{9} \mathrm{Be}$ data are from experiments ACE [47], Balloon [48-50], IMP7\&8 [51], ISEE3-HKH [52], ISOMAX [53], Ulysses-HET [54] and Voyager [55].

TABLE II: The nucleon injection parameters derived through fitting the proton data of AMS-02. A single power-law is enough to fit the data in the DC scenario.

\begin{tabular}{lccl}
\hline \hline & Prior Range & DR & DC \\
\hline$\nu_{1}$ & {$[1.0,4.0]$} & $1.811 \pm 0.021$ & $2.336 \pm 0.004$ \\
$\nu_{2}$ & {$[1.0,4.0]$} & $2.402 \pm 0.005$ & $2.336 \pm 0.004$ \\
$R_{\mathrm{br}}^{p}(\mathrm{GV})$ & {$[8.0,15.0]$} & $12.88 \pm 0.263$ & 10.00 \\
$A_{p}{ }^{a}$ & {$[3.0,6.0]$} & $4.613 \pm 0.027$ & $4.783 \pm 0.026$ \\
$\phi_{p}(\mathrm{MV})$ & {$[50,1500]$} & $517.8 \pm 37.8$ & $505.9 \pm 13.1$ \\
\hline \hline
\end{tabular}

${ }^{a}$ Post-propagated normalization flux of protons at $100 \mathrm{GeV}$ in unit $10^{-9} \mathrm{~cm}^{-2} \mathrm{~s}^{-1} \mathrm{sr}^{-1} \mathrm{MeV}^{-1}$

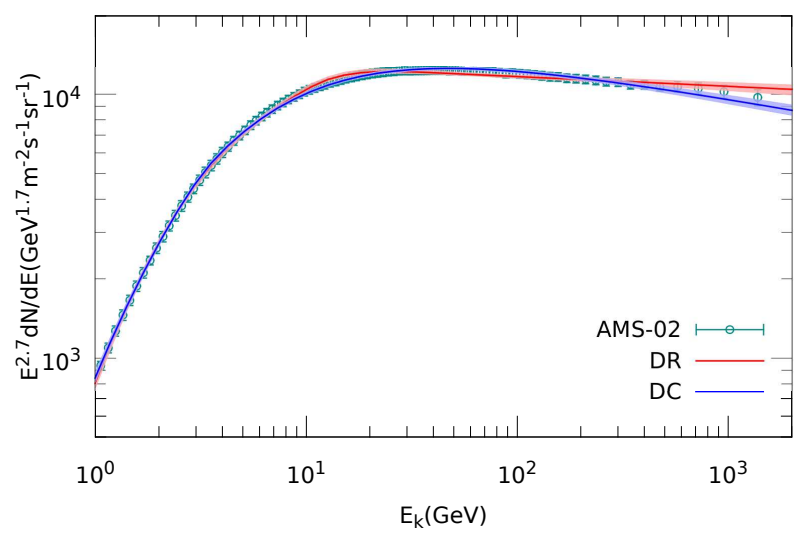

FIG. 2: The fluxes of protons for the corresponding parameter shown in Table II, compared with the preliminary data from AMS-02 [38]. The bands delimit the regions of $95 \%$ confidence level.

About the primary electrons, we also assume a broken power-law form of the injection spectrum. Since electrons lose energies much more efficiently than the nuclei, the effect from recent and nearby sources may make the spectrum fluctuate significantly, especially at high energies [12]. Therefore there might be more structures in the electron spectrum. We will discuss two cases in this work: one break case similar to Eq. (4), and three-piece broken power law with two breaks. The latter is found to be required to fit the pre-AMS-02 lepton data [10, 37, 64, 65]. Thus the electron injection parameters are

with one break: $\left(\gamma_{1}, \gamma_{2}, R_{\mathrm{br}}^{e}, A_{e}\right)$; with two breaks: $\left(\gamma_{1}, R_{\mathrm{br}}^{e}, \gamma_{2}, R_{\mathrm{br} 2}^{e}, \gamma_{3}, A_{e}\right)$.

\section{THE PARAMETERS FOR THE EXTRA SOURCES}

In this paper, two kinds of extra source, including pulsars and the DM annihilation, will be discussed. The pulsars are able to generate high energy positron-electron pairs through the electromagnetic cascade in the magnetic pole region, which could cause the observed excess [15-19]. The injection spectrum of the electrons and positrons is usually assumed to be a power law with an exponential cutoff

$$
q_{e}^{\mathrm{psr}}=A_{\mathrm{psr}}(R / \mathrm{MV})^{-\alpha} \exp \left(-R / R_{\mathrm{c}}\right),
$$

where $A_{\mathrm{psr}}$ is the normalization factor, $\alpha$ is the spectral index, $R_{\mathrm{c}}$ is the cutoff rigidity. We adopt a continuous and stable pulsar injection. The spatial distribution obeys the same form of Eq. (3), with slightly different parameters $a=2.35$ and $b=5.56$ [66].

The particle and antiparticle of DM in the Galaxy, if the interaction is strong enough, can also annihilate with each other and produce standard model particles which are injected in the Galaxy as CRs. Since there is no obvious excess of antiprotons from the secondary expectation during the CR propagation compared with the data [67], 
leptonical annihilation final states are expected [25, 26]. We therefore discuss the model with annihilation final states of a pair of muons or tauons. We use the results of PPPC 4 DM ID [68], which includes the electroweak corrections [69], to calculate the electron (positron) spectrum from DM annihilation. The Navarro-Frenk-White (NFW) density profile [70]

$$
\rho(r)=\frac{\rho_{s}}{\left(r / r_{s}\right)\left(1+r / r_{s}\right)^{2}},
$$

where $\rho_{s}=20 \mathrm{kpc}$ and $\rho_{s}=0.26 \mathrm{GeV} \mathrm{cm}^{-3}$, is adopted to describe the spatial distribution of DM in the Milky Way halo. The free parameters in the DM annihilation scenario include the DM particle mass $m_{\chi}$ and the velocityweighted average annihilation cross section $\langle\sigma v\rangle$.

\section{THE FITTING RESULTS}

The datasets used in this study include the latest measurements of the positron fraction $e^{+} /\left(e^{+}+e^{-}\right)$, fluxes of $e^{-}, e^{+}$and $\left(e^{+}+e^{-}\right)$by AMS-02 [39-41]. These data may not be fully uncorrelated, since the positron fraction may be derived from the fluxes of $e^{-}$and $e^{+}$. However, the analysis methods are different for various kinds of measurements, and the systematic uncertainties also differ from one to another. Therefore we adopted all these data in the study, though the statistics of the data would be over estimated. We have also tested that when dropping one group of the data the results are almost unchanged. We further select the data above $1 \mathrm{GeV}$, since the lowest energy data may be significantly affected by the solar modulation and may not be well modeled in the force-field approximation [57]. The spectral index of electrons/positrons injected by pulsars, and possibly altered by the surrounding pulsar wind nebulae, is actually very uncertain. In this work we limit $\alpha$ to the range between 1.0 and 2.4 , according to the radio and $\gamma$-ray observations of pulsars [71-73].

\section{A. One break in primary electron spectrum}

In this case there are 4 parameters of the primary electrons. Together with $c_{e^{+}}, \phi$ and the extra source parameters, we have in total 9 (8) parameters for the pulsar (DM) scenario. The best-fitting parameters and the mean values as well as $1 \sigma$ confidence ranges are shown in Tables III, IV and V for the pulsar, DM annihilation into $\mu^{+} \mu^{-}$and DM annihilation into $\tau^{+} \tau^{-}$respectively. Figs. 3 and 4 illustrate the comparison of the best-fitting results with the data, for DR and DC propagation scenarios. Table VI summarizes the fitting $\chi^{2}$ values for each dataset.

The results show not good enough fittings to data. From Figs. 3 and 4 it can be seen that while the model may over-produce the positron fraction, it is not enough to reproduce the electron flux at high energies. This is similar to that we found before using the electron data from PAMELA/Fermi-LAT [9]. The fitting $\chi^{2}$ values also show this issue. The minimum $\chi^{2}$ value for these 6 fittings is 374.2 , and the reduced $\chi^{2}$ is about 1.42 for 264 degree of freedom (d.o.f.). It corresponds to a $4.4 \sigma$ deviation from a good fitting as expected.

Given the fittings are poor, the constraints on the model parameters by minimizing the $\chi^{2}$ may not be physically meaningful. The pulsar model gives better fitting than the DM scenario, since the spectral index of electrons/positrons injected by pulsar is enabled to vary and it has larger d.o.f. compared with the spectrum expected from DM annihilation. We further note that the $\chi^{2}$ contributed from the $e^{-}$flux and the $e^{+}+e^{-}$flux are about two times larger than that from $e^{+}$flux, although the numbers of data points are comparable. It could be due to the fact that electrons have much higher statistics compared with the positrons. The failure to reproduce the high energy electron spectrum well would result in a large $\chi^{2}$ value. Therefore, we may need to change the background model to improve the fitting of the high energy electron spectrum.

\section{B. Two breaks in primary electron spectrum}

A direct way to alleviate the tension shown above is to add more electrons at high energies, such as a spectral hardening $[10,64,65]$. For the nuclei spectra similar spectral hardening above several hundred GV has been observed by ATIC [74], CREAM [75] and PAMELA [76], and could be naturally expected if there is a diversity of the source parameters [77]. Therefore we apply a second break on the injection spectrum of the primary electrons characterized by two additional parameters $\gamma_{3}$ and $R_{\mathrm{br} 2}^{e}$. The fitting results are shown in Tables VII, VIII, IX and Figs. 5, 6 respectively. We also show in Figs. 7 and 8 the derived one and two dimentional posterior distributions of the most relevant parameters, choosing the DM annihilation into $\mu$ channel as benchmark cases.

Significant improvements of the fittings can be seen from these results. It is shown from Table VI that in all the cases the reduced $\chi^{2}$ values are about 2 times smaller than the previous case with one break. For most cases the reduced $\chi^{2}$ is close to or smaller than 1 . Since the systematic errors are added quadratically to the statistical errors to calculate the $\chi^{2}$, it is expected that the reduced $\chi^{2}$ value will be smaller than 1 if the model does fit the data well (see e.g. the minimum model of [40]). It can be seen from Figs. 5 and 6 that the contribution of positrons from the extra source dominates over the secondary component above $\sim 50-100 \mathrm{GeV}$. For electrons, however, the background component will always dominate in the energy range from $1 \mathrm{GeV}$ to $1 \mathrm{TeV}$.

We further find that the pulsar model and DM annihilation into $\tau^{+} \tau^{-}$give comparable fittings to the data. However, the case for $\mu^{+} \mu^{-}$channel seems to be more 
TABLE III: Fitting results of pulsar model with one break in $e^{-}$injection spectrum

\begin{tabular}{lcrrrrr}
\hline & \multirow{2}{*}{ Prior Range } & \multicolumn{2}{c}{ DR } & & \multicolumn{2}{c}{ DC } \\
\cline { 3 - 4 } \cline { 5 - 6 } & & Best & Mean & & Best & Mean \\
\hline $\log \left(A_{e}{ }^{a}\right)$ & {$[-10.5,-7.5]$} & -8.812 & $-8.813 \pm 0.002$ & & -8.897 & $-8.897 \pm 0.002$ \\
$\gamma_{1}$ & {$[1.0,3.0]$} & 1.896 & $1.878 \pm 0.025$ & & 2.268 & $2.264 \pm 0.02$ \\
$\gamma_{2}$ & {$[1.5,4.0]$} & 2.874 & $2.872 \pm 0.005$ & & 2.725 & $2.726 \pm 0.005$ \\
$\log \left(R_{\mathrm{br}}^{e} / \mathrm{MV}\right)$ & {$[3.0,6.0]$} & 3.697 & $3.68 \pm 0.025$ & & 3.812 & $3.808 \pm 0.014$ \\
$\log \left(A_{\mathrm{psr}}^{b}\right)$ & {$[-35.0,-20.0]$} & -25.99 & $-25.91 \pm 0.21$ & & -25.31 & $-25.35 \pm 0.12$ \\
$\alpha$ & {$[1.0,2.4]$} & 1.749 & $1.763 \pm 0.039$ & & 1.859 & $1.851 \pm 0.022$ \\
$\log \left(R_{\mathrm{c}} / \mathrm{MeV}\right)$ & {$[4.0,10.0]$} & 9.621 & $8.88 \pm 0.7$ & & 9.168 & $8.782 \pm 0.77$ \\
$c_{e}+$ & {$[0.25,4.0]$} & 2.63 & $2.599 \pm 0.057$ & & 1.204 & $1.216 \pm 0.033$ \\
$\phi / \mathrm{MV}$ & {$[100,1500]$} & 1410.0 & $1402.0 \pm 13.0$ & 562.1 & $565.9 \pm 12.0$ \\
\hline
\end{tabular}

${ }^{a}$ Post-propagated normalization flux of $e^{-}$at $25 \mathrm{GeV}$ in unit $\mathrm{cm}^{-2} \mathrm{~s}^{-1} \mathrm{sr}^{-1} \mathrm{MeV}^{-1}$.

${ }^{b}$ Pre-propagated normalization of pulsar injection at $1 \mathrm{MeV}$ in unit $\mathrm{cm}^{-3} \mathrm{~s}^{-1} \mathrm{MeV}^{-1}$.

TABLE IV: Fitting results of DM annihilation scenario in $\mu^{+} \mu^{-}$channel with one break in $e^{-}$injection spectrum

\begin{tabular}{lcrrrrr}
\hline & \multirow{2}{*}{ Prior Range } & \multicolumn{2}{c}{ DR } & & \multicolumn{2}{c}{ DC } \\
\cline { 3 - 4 } \cline { 6 - 6 } & & \multicolumn{1}{c}{ Best } & \multicolumn{1}{c}{ Mean } & & Best & \multicolumn{1}{c}{ Mean } \\
\hline $\log \left(A_{e}{ }^{a}\right)$ & {$[-10.5,-7.5]$} & -8.801 & $-8.801 \pm 0.001$ & & -8.872 & $-8.872 \pm 0.002$ \\
$\gamma_{1}$ & {$[1.0,3.0]$} & 1.925 & $1.913 \pm 0.018$ & & 2.303 & $2.301 \pm 0.037$ \\
$\gamma_{2}$ & {$[1.5,4.0]$} & 2.9 & $2.9 \pm 0.003$ & & 2.79 & $2.789 \pm 0.004$ \\
$\log \left(R_{\mathrm{br}}^{e} / \mathrm{MV}\right)$ & {$[3.0,6.0]$} & 3.706 & $3.692 \pm 0.019$ & & 3.702 & $3.704 \pm 0.016$ \\
$\log \left(m_{\chi} / \mathrm{GeV}\right)$ & {$[0.0,7.0]$} & 3.179 & $3.178 \pm 0.066$ & & 2.654 & $2.65 \pm 0.018$ \\
$\log \left(\langle\sigma v\rangle^{b}\right)$ & {$[-28.0,-18.0]$} & -22.85 & $-22.86 \pm 0.12$ & & -23.42 & $-23.43 \pm 0.029$ \\
$c_{e}$ & {$[0.25,4.0]$} & 2.997 & $2.994 \pm 0.019$ & & 1.775 & $1.771 \pm 0.014$ \\
$\phi / \mathrm{MV}$ & {$[100,1500]$} & 1488.0 & $1488.0 \pm 6.7$ & & 748.2 & $744.8 \pm 7.9$ \\
\hline
\end{tabular}

${ }^{a}$ Post-propagated normalization flux of $e^{-}$at $25 \mathrm{GeV}$ in unit $\mathrm{cm}^{-2} \mathrm{~s}^{-1} \mathrm{sr}^{-1} \mathrm{MeV}^{-1}$.

${ }^{b}$ In unit $\mathrm{cm}^{3} \mathrm{~s}^{-1}$

TABLE V: Fitting results of DM annihilation scenario in $\tau^{+} \tau^{-}$channel with one break in $e^{-}$injection spectrum

\begin{tabular}{lcrcrrr}
\hline & \multirow{2}{*}{ Prior Range } & \multicolumn{3}{c}{ DR } & & \multicolumn{2}{c}{ DC } \\
\cline { 3 - 4 } \cline { 6 - 7 } & & \multicolumn{1}{c}{ Best } & Mean & & Best & Mean \\
\hline $\log \left(A_{e}{ }^{a}\right)$ & {$[-10.5,-7.5]$} & -8.803 & $-8.803 \pm 0.002$ & & -8.883 & $-8.882 \pm 0.002$ \\
$\gamma_{1}$ & {$[1.0,3.0]$} & 1.915 & $1.909 \pm 0.019$ & & 2.299 & $2.295 \pm 0.031$ \\
$\gamma_{2}$ & {$[1.5,4.0]$} & 2.895 & $2.895 \pm 0.004$ & & 2.757 & $2.758 \pm 0.004$ \\
$\log \left(R_{\mathrm{br}}^{e} / \mathrm{MV}\right)$ & {$[3.0,6.0]$} & 3.698 & $3.691 \pm 0.02$ & & 3.728 & $3.722 \pm 0.015$ \\
$\log \left(m_{\chi} / \mathrm{GeV}\right)$ & {$[0.0,7.0]$} & 3.78 & $3.786 \pm 0.12$ & & 2.936 & $2.954 \pm 0.023$ \\
$\log \left(\langle\sigma v\rangle^{b}\right)$ & {$[-28.0,-18.0]$} & -21.72 & $-21.71 \pm 0.2$ & & -22.72 & $-22.69 \pm 0.034$ \\
$c_{e}$ & {$[0.25,4.0]$} & 2.934 & $2.942 \pm 0.026$ & & 1.592 & $1.601 \pm 0.016$ \\
$\phi / \mathrm{MV}$ & {$[100,1500]$} & 1472.0 & $1475.0 \pm 8.8$ & & 670.8 & $674.9 \pm 8.7$ \\
\hline
\end{tabular}

${ }^{a}$ Post-propagated normalization flux of $e^{-}$at $25 \mathrm{GeV}$ in unit $\mathrm{cm}^{-2} \mathrm{~s}^{-1} \mathrm{sr}^{-1} \mathrm{MeV}^{-1}$.

${ }^{b}$ In unit $\mathrm{cm}^{3} \mathrm{~s}^{-1}$

TABLE VI: Fitting $\chi^{2}$ values and the contribution from each data. The number of data points for $e^{+} /\left(e^{+}+e^{-}\right), e^{+}+e^{-}, e^{-}$ and $e^{+}$are $63,71,70$ and 69 , respectively.

\begin{tabular}{|c|c|c|c|c|c|c|c|c|c|c|c|c|c|}
\hline & & \multicolumn{5}{|c|}{ two breaks } & \multirow[b]{2}{*}{$e^{+}$} & \multicolumn{5}{|c|}{ one break } & \multirow[b]{2}{*}{$e^{+}$} \\
\hline & & $\frac{\chi^{2}}{\text { d.o.f. }}$ & $\chi^{2}$ & $\frac{e^{+}}{e^{+}+e^{-}}$ & $e^{+}+e^{-}$ & $e^{-}$ & & $\frac{\chi^{2}}{\text { d.o.f. }}$ & $\chi^{2}$ & $\frac{e^{+}}{e^{+}+e^{-}}$ & $e^{+}+e^{-}$ & $e^{-}$ & \\
\hline \multirow{3}{*}{ DR } & $\overline{\mathrm{PSR}}$ & 1.1 & 287.1 & 48.57 & 99.02 & 60.03 & 79.45 & 2.75 & 725.2 & 132.7 & 271.8 & 211.7 & 109.0 \\
\hline & $\mu$ & 1.12 & 293.4 & 40.7 & 113.8 & 61.0 & 77.95 & 3.01 & 797.9 & 242.4 & 245.5 & 206.9 & 103.1 \\
\hline & $\tau$ & 1.11 & 291.0 & 42.62 & 106.4 & 63.38 & 78.6 & 2.94 & 779.9 & 213.9 & 252.2 & 209.9 & 103.9 \\
\hline \multirow{3}{*}{$\mathrm{DC}$} & PSR & 0.411 & 107.6 & 51.58 & 17.33 & 17.62 & 21.07 & 1.42 & 374.2 & 89.04 & 121.3 & 119.1 & 44.78 \\
\hline & $\mu$ & 1.27 & 334.8 & 116.1 & 90.8 & 35.97 & 91.93 & 3.95 & 1048.0 & 484.1 & 250.0 & 179.8 & 134.1 \\
\hline & $\tau$ & 0.575 & 151.1 & 65.0 & 30.18 & 17.97 & 37.91 & 2.4 & 636.5 & 168.5 & 228.1 & 165.1 & 74.84 \\
\hline
\end{tabular}



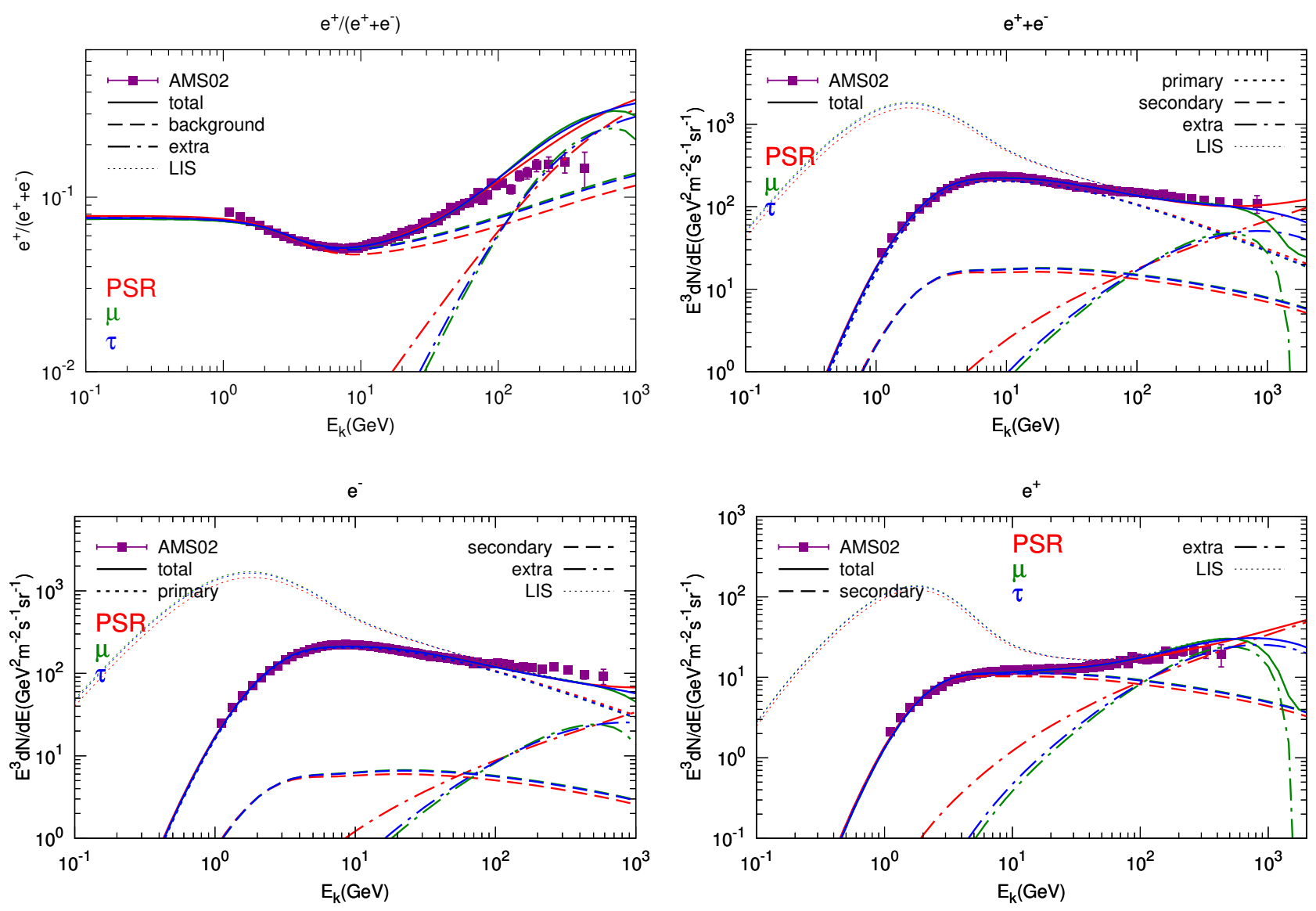

FIG. 3: The expected results for the best-fitting parameters in DR scenario with one-break of the primary electron injection spectrum. Top-left: positron fraction $e^{+} /\left(e^{+}+e^{-}\right)$; top-right: electron plus positron flux $e^{+}+e^{-}$; bottom-left: electron flux $e^{-}$; bottom-right: positron flux $e^{+}$. The red, green and blue curves represent the pulsar, DM annihilation into $\mu^{+} \mu^{-}$and $\tau^{+} \tau^{-}$ final states, respectively. Different line styles represent different components as labeled.

TABLE VII: Fitting results of pulsar model with two breaks in $e^{-}$injection spectrum

\begin{tabular}{lcrcccc}
\hline & \multirow{2}{*}{ Prior Range } & \multicolumn{3}{c}{ DR } & & \multicolumn{2}{c}{ DC } \\
\cline { 3 - 4 } \cline { 6 - 7 } $\log \left(A_{e}{ }^{a}\right)$ & {$[-10.5,-7.5]$} & -8.813 & $-8.812 \pm 0.002$ & & -8.896 & $-8.897 \pm 0.002$ \\
$\gamma_{1}$ & {$[1.0,3.0]$} & 1.302 & $1.361 \pm 0.054$ & & 2.382 & $2.377 \pm 0.022$ \\
$\log \left(R_{\mathrm{br}}^{e} / \mathrm{MV}\right)$ & {$[3.0,5.0]$} & 3.406 & $3.42 \pm 0.02$ & & 3.881 & $3.873 \pm 0.02$ \\
$\gamma_{2}$ & {$[1.5,4.0]$} & 2.976 & $2.972 \pm 0.005$ & & 2.836 & $2.829 \pm 0.011$ \\
$\log \left(R_{\mathrm{br} 2}^{e} / \mathrm{MV}\right)$ & {$[4.0,6.0]$} & 4.778 & $4.794 \pm 0.028$ & & 4.717 & $4.747 \pm 0.044$ \\
$\gamma_{3}$ & {$[1.5,4.0]$} & 2.668 & $2.656 \pm 0.02$ & & 2.586 & $2.571 \pm 0.02$ \\
$\log \left(A_{\mathrm{psr}}{ }^{b}\right)$ & {$[-35.0,-20.0]$} & -28.88 & $-28.71 \pm 0.59$ & & -26.8 & $-26.77 \pm 0.46$ \\
$\alpha$ & {$[1.0,2.4]$} & 1.185 & $1.221 \pm 0.12$ & & 1.564 & $1.569 \pm 0.096$ \\
$\log \left(R_{\mathrm{c}} / \mathrm{MeV}\right)$ & {$[4.0,10.0]$} & 5.853 & $5.923 \pm 0.22$ & & 6.073 & $6.087 \pm 0.23$ \\
$c_{e}+$ & {$[0.25,4.0]$} & 3.029 & $3.02 \pm 0.025$ & & 1.53 & $1.512 \pm 0.048$ \\
$\phi / \mathrm{MV}$ & {$[100,1500]$} & 1499.0 & $1495.0 \pm 4.4$ & & 672.8 & $667.3 \pm 16.0$ \\
\hline
\end{tabular}

${ }^{a}$ Post-propagated normalization flux of $e^{-}$at $25 \mathrm{GeV}$ in unit $\mathrm{cm}^{-2} \mathrm{~s}^{-1} \mathrm{sr}^{-1} \mathrm{MeV}^{-1}$.

${ }^{b}$ Pre-propagated normalization of pulsar injection at $1 \mathrm{MeV}$ in unit $\mathrm{cm}^{-3} \mathrm{~s}^{-1} \mathrm{MeV}^{-1}$. 

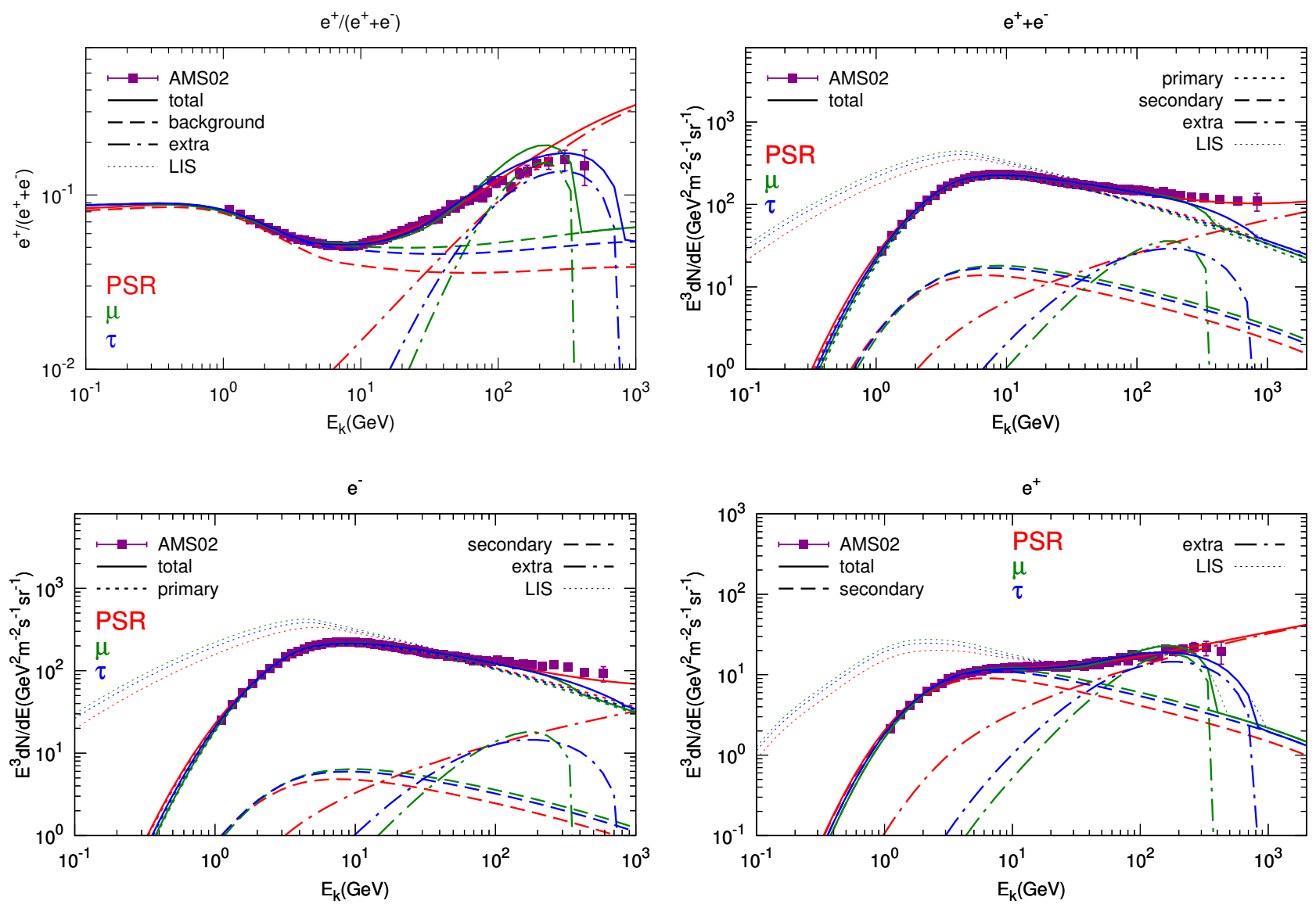

FIG. 4: Same as Fig. 3 but for DC propagation scenario.

TABLE VIII: Fitting results of DM annihilation scenario in $\mu^{+} \mu^{-}$channel with two breaks in $e^{-}$injection spectrum

\begin{tabular}{lcrrrrr}
\hline & \multirow{2}{*}{ Prior Range } & \multicolumn{2}{c}{ DR } & & \multicolumn{2}{c}{ DC } \\
\cline { 3 - 4 } \cline { 6 - 7 } & & \multicolumn{1}{c}{ Best } & \multicolumn{1}{c}{ Mean } & & Best & \multicolumn{1}{c}{ Mean } \\
\hline $\log \left(A_{e}{ }^{a}\right)$ & {$[-10.5,-7.5]$} & -8.813 & $-8.812 \pm 0.001$ & & -8.887 & $-8.888 \pm 0.002$ \\
$\gamma_{1}$ & {$[1.0,3.0]$} & 1.501 & $1.53 \pm 0.028$ & & 2.427 & $2.427 \pm 0.022$ \\
$\log \left(R_{\mathrm{br}}^{e} / \mathrm{MV}\right)$ & {$[3.0,5.0]$} & 3.473 & $3.48 \pm 0.013$ & & 3.849 & $3.847 \pm 0.016$ \\
$\gamma_{2}$ & {$[1.5,4.0]$} & 2.976 & $2.971 \pm 0.005$ & & 2.894 & $2.896 \pm 0.01$ \\
$\log \left(R_{\mathrm{br} 2}^{e} / \mathrm{MV}\right)$ & {$[4.0,6.0]$} & 4.787 & $4.803 \pm 0.029$ & & 4.712 & $4.713 \pm 0.038$ \\
$\gamma_{3}$ & {$[1.5,4.0]$} & 2.654 & $2.647 \pm 0.021$ & & 2.558 & $2.553 \pm 0.023$ \\
$\log \left(m_{\chi} / \mathrm{GeV}\right)$ & {$[0.0,7.0]$} & 2.964 & $2.957 \pm 0.049$ & & 2.621 & $2.621 \pm 0.02$ \\
$\log \left(\langle\sigma v\rangle^{b}\right)$ & {$[-28.0,-18.0]$} & -23.3 & $-23.31 \pm 0.085$ & & -23.53 & $-23.53 \pm 0.032$ \\
$c_{e}+$ & {$[0.25,4.0]$} & 3.053 & $3.049 \pm 0.013$ & & 1.855 & $1.858 \pm 0.015$ \\
$\phi / \mathrm{MV}$ & {$[100,1500]$} & 1500.0 & $1498.0 \pm 2.2$ & & 781.8 & $784.8 \pm 8.2$ \\
\hline
\end{tabular}

${ }^{a}$ Post-propagated normalization flux of $e^{-}$at $25 \mathrm{GeV}$ in unit $\mathrm{cm}^{-2} \mathrm{~s}^{-1} \mathrm{sr}^{-1} \mathrm{MeV}^{-1}$.

${ }^{b}$ In unit $\mathrm{cm}^{3} \mathrm{~s}^{-1}$

complicated. In the DR scenario it gives comparable fittings compared with the pulsar model and the DM annihilation into $\tau^{+} \tau^{-}$channel, while in the DC scenario, the fitting results become worse. Fig. 9 shows the comparison of the positron fluxes between DR and DC scenarios, for DM annihilation into $\mu^{+} \mu^{-}$channel. The reason of such a result might be the difference of the secondary positron spectrum in the two scenarios. As can be seen from Fig. 9 the secondary positron spectrum is softer in the DC scenario. This should be due to that the DC model has a larger propagation parameter $\delta$ than the DR model. Since the positron spectrum from muon decay is very hard, a smaller value of DM mass is needed to better fit the data. It results in the failure to reproduce the 
TABLE IX: Fitting results of DM annihilation scenario in $\tau^{+} \tau^{-}$channel with two breaks in $e^{-}$injection spectrum

\begin{tabular}{lcrrrrr}
\hline & \multirow{2}{*}{ Prior Range } & \multicolumn{3}{c}{ DR } & & \multicolumn{2}{c}{ DC } \\
\cline { 3 - 4 } \cline { 6 - 7 } & & \multicolumn{1}{c}{ Best } & \multicolumn{1}{c}{ Mean } & & Best & Mean \\
\hline $\log \left(A_{e}{ }^{a}\right)$ & {$[-10.5,-7.5]$} & -8.813 & $-8.812 \pm 0.002$ & & -8.891 & $-8.891 \pm 0.002$ \\
$\gamma_{1}$ & {$[1.0,3.0]$} & 1.502 & $1.528 \pm 0.025$ & & 2.409 & $2.402 \pm 0.021$ \\
$\log \left(R_{\mathrm{br}}^{e} / \mathrm{MV}\right)$ & {$[3.0,5.0]$} & 3.471 & $3.48 \pm 0.012$ & & 3.842 & $3.84 \pm 0.015$ \\
$\gamma_{2}$ & {$[1.5,4.0]$} & 2.972 & $2.969 \pm 0.005$ & & 2.855 & $2.856 \pm 0.009$ \\
$\log \left(R_{\mathrm{br} 2}^{e} / \mathrm{MV}\right)$ & {$[4.0,6.0]$} & 4.789 & $4.803 \pm 0.029$ & & 4.756 & $4.746 \pm 0.04$ \\
$\gamma_{3}$ & {$[1.5,4.0]$} & 2.656 & $2.651 \pm 0.021$ & & 2.548 & $2.555 \pm 0.021$ \\
$\log \left(m_{\chi} / \mathrm{GeV}\right)$ & {$[0.0,7.0]$} & 3.59 & $3.581 \pm 0.081$ & & 3.003 & $3.006 \pm 0.028$ \\
$\log \left(\langle\sigma v\rangle^{b}\right)$ & {$[-28.0,-18.0]$} & -22.13 & $-22.14 \pm 0.14$ & & -22.68 & $-22.67 \pm 0.039$ \\
$c_{e}+$ & {$[0.25,4.0]$} & 3.035 & $3.027 \pm 0.015$ & & 1.719 & $1.727 \pm 0.018$ \\
$\phi / \mathrm{MV}$ & {$[100,1500]$} & 1500.0 & $1496.0 \pm 3.1$ & & 728.9 & $732.6 \pm 9.3$ \\
\hline
\end{tabular}

${ }^{a}$ Post-propagated normalization flux of $e^{-}$at $25 \mathrm{GeV}$ in unit $\mathrm{cm}^{-2} \mathrm{~s}^{-1} \mathrm{sr}^{-1} \mathrm{MeV}^{-1}$

${ }^{b}$ In unit $\mathrm{cm}^{3} \mathrm{~s}^{-1}$
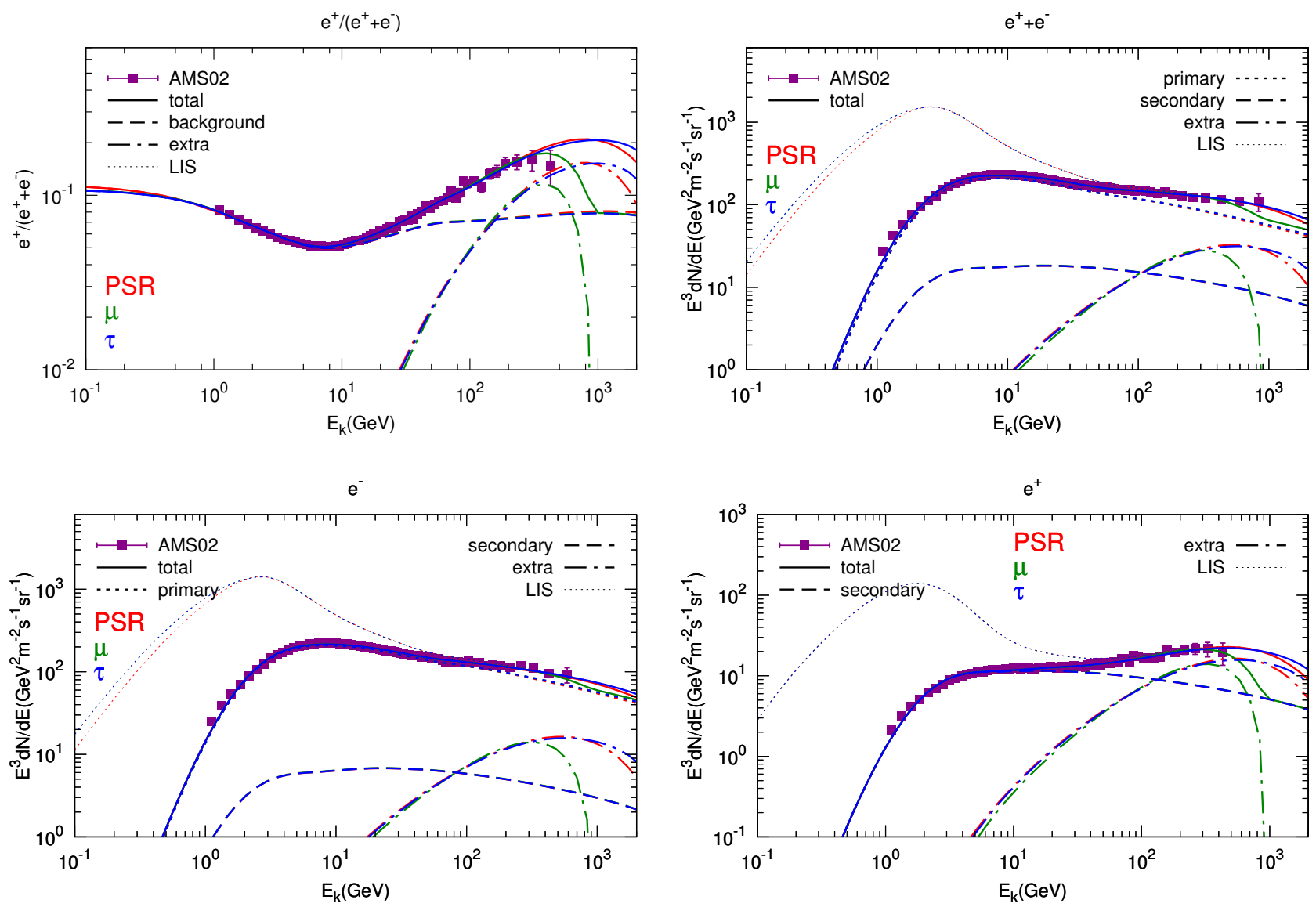

FIG. 5: The same as Fig. 3 but for the background model with two breaks in the electron injection spectrum.

high energy tail of the positron spectrum. The positron spectrum from pulsar or tauon decay can be softer, thus can fit the data better than the muon model ${ }^{2}$.

\footnotetext{
${ }^{2}$ A four-muon final state may give better fitting to the AMS-02 data.
} 

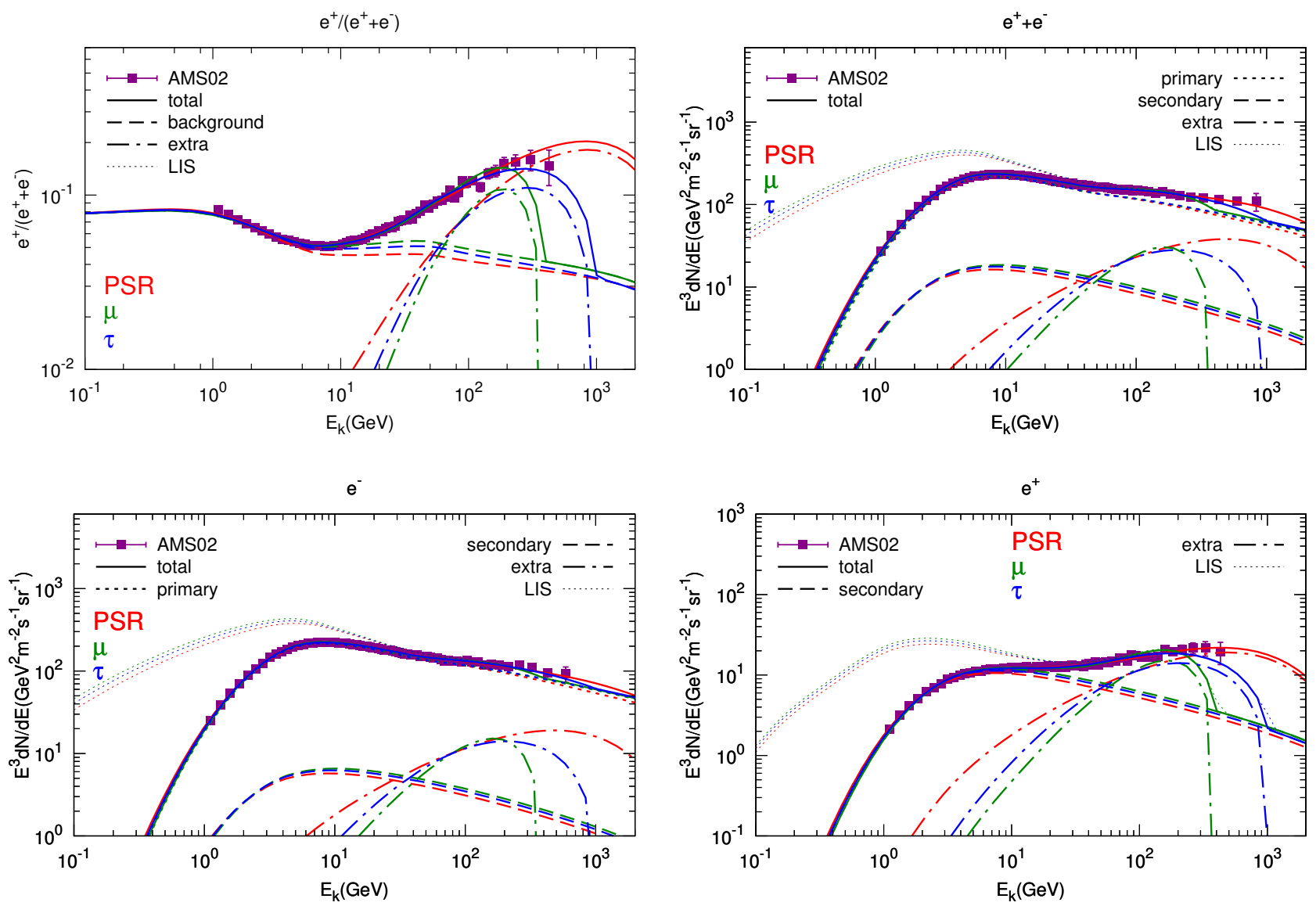

FIG. 6: The same as Fig. 5 but for DC propagation scenario.

\section{DISCUSSION}

In this paper we try to give a quantitative study on the AMS-02 results of the electron/positron fluxes. Although the AMS-02 data are precise enough, there are large uncertainties from the theoretical model parameters, such as the uncertainties from the $\mathrm{CR}$ propagation model, the treatment of the solar modulation, the Galactic gas distribution and so on. In [37], we studied quite a few such kinds of uncertainties as possible systematical uncertainties, including the propagation, the solar modulation and low energy data selection, the hadronic interaction model and so on. The study shows that although the uncertainties of the model inputs seem to be large, the fitting results about the extra sources are under good control.

As an illustration, we plot the $1 \sigma$ and $2 \sigma$ contours on the $m_{\chi}-\langle\sigma v\rangle$ parameter plane to show the uncertainties of the parameter determination for the scenarios discussed in this work. The solid (dashed) ones are for the case with two (one) breaks of the primary electron injection spectrum. The red ones are for DR propagation model and blues ones are for DC model. The results do show some differences, between DR and DC propagation models. Nevertheless, the shift of the central values as well as the contours is about a factor of 4 , which is larger than that found in [37]. One possible reason for this difference might be that we do not include the HESS data at higher energies in this study. The HESS data, although have large systematic uncertainties, should be useful to constrain the very high energy behavior of the electron/positron spectra. The future experiments such as DAMPE ${ }^{3}$ and HERD [78] may provide better measurements of the electron/positron spectra above $\mathrm{TeV}$.

A main result of our fitting is that a new feature at the primary electron spectrum is strongly favored. Such a feature indicates that the nearby and/or fresh CR sources may contribute to the high energy electrons with a harder spectrum than the background [12]. Considering the large fluctuation of the electron/positron fluxes in space due to fast energy losses it is quite reasonable that the high energy electrons are dominated by the local sources. One possible signature of such a scenario may be the fine structures of the electron/positron spectra which may be

\footnotetext{
${ }^{3}$ http://dpnc.unige.ch/dampe/
} 


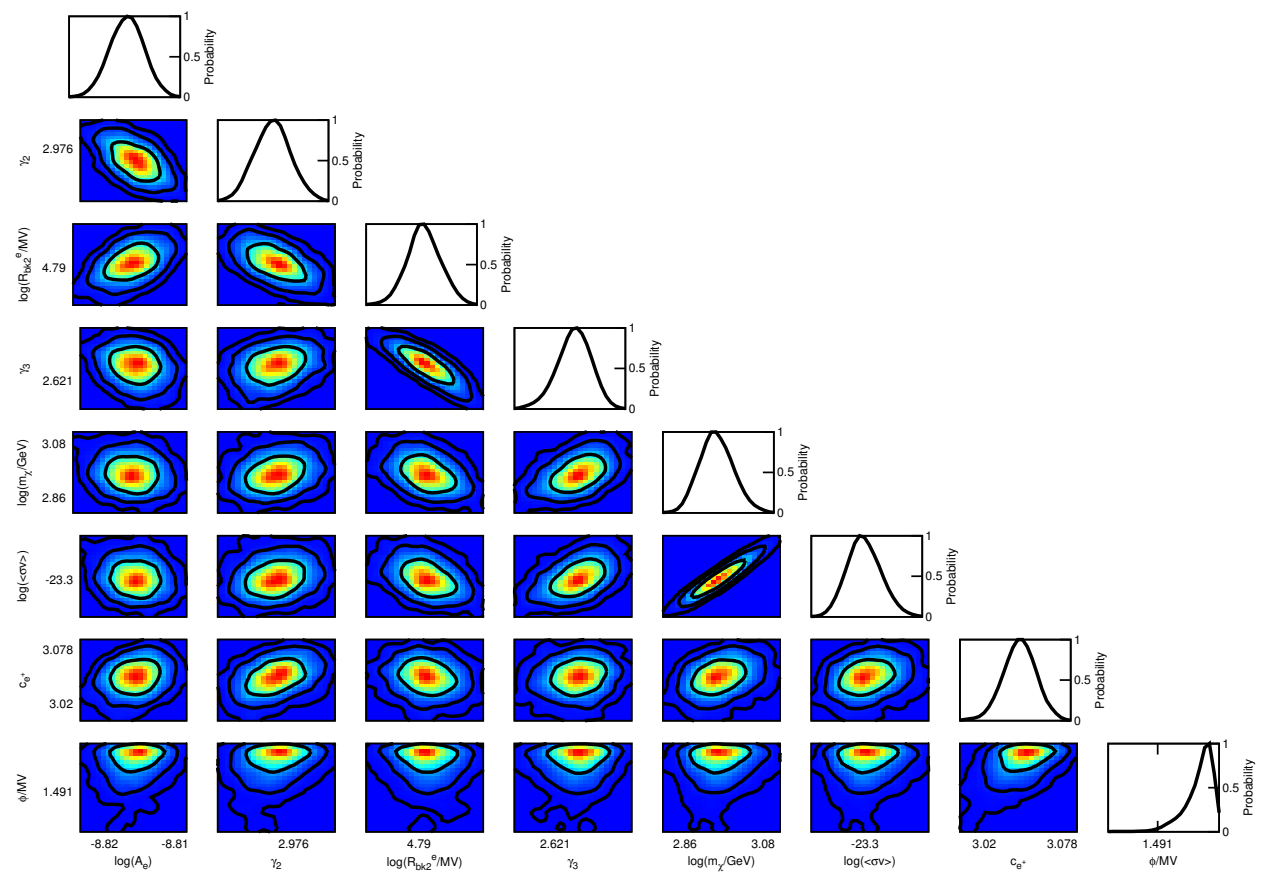

FIG. 7: The 1 and 2-dimensional distributions of part of the parameters for DM annihilation into $\mu^{+} \mu^{-}$channel. The background electrons have two breaks, and the propagation model is DR. The contours in the 2-dimensional plots denote the $68.3 \%, 95.5 \%$ and $99.7 \%$ confidence levels from inside to outside.

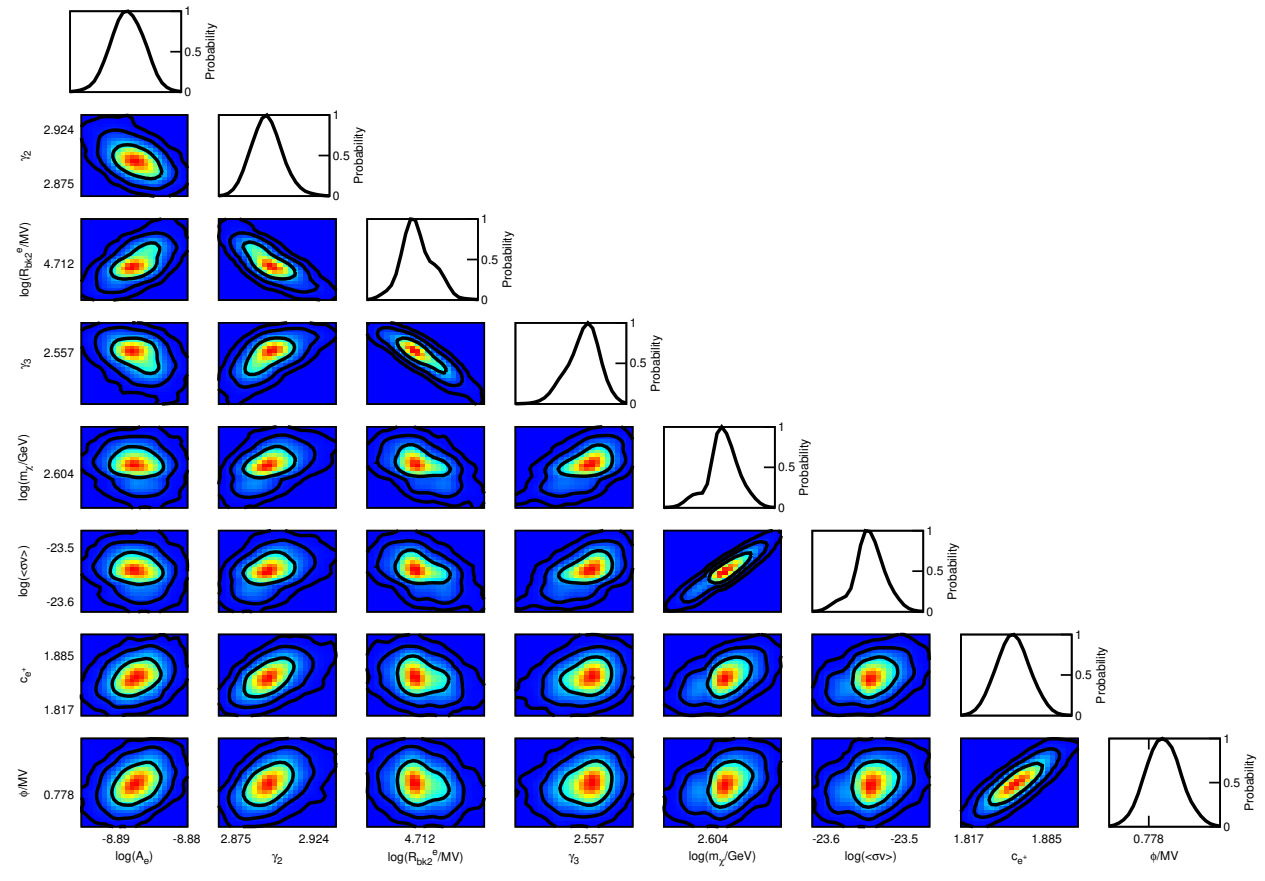

FIG. 8: The same as Fig. 7, but for the DC propagation scenario.

investigated with future observations [79]. Another possible probe of the local sources could be the anisotropy measurements of the electrons [80].

Another interesting conclusion of this study is that in general the DC model is more favored than the DR model by the lepton spectra (Table VI). The DM model with $\mu^{+} \mu^{-}$channel is an exception which we have discussed above. The reason is that the local interstellar (LIS) spectrum has a bump at low energy in the DR model due to the reacceleration, and thus a large solar modulation 


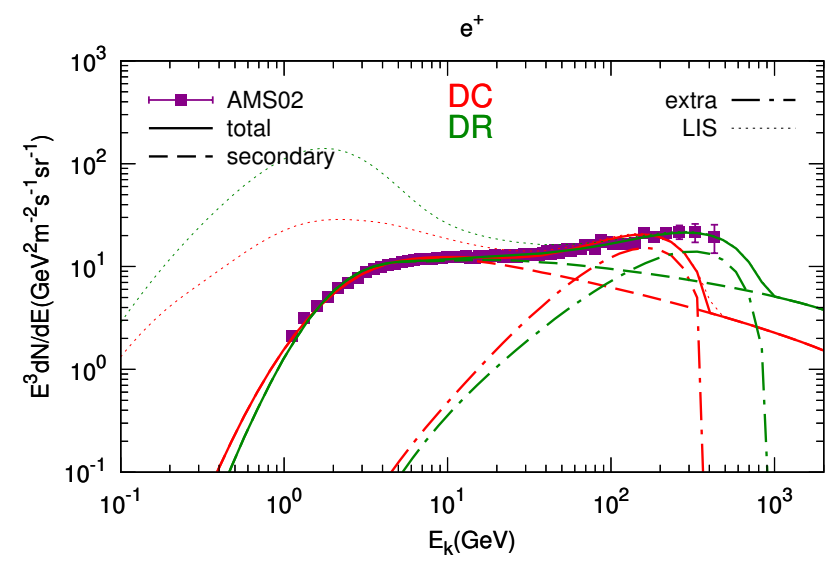

FIG. 9: Comparison of the positron spectra between the DC scenario (red) and DR scenario (green) with extra source component from DM annihilation into $\mu^{+} \mu^{-}$final state.

potential has to be introduced to suppress the bump to fit the data. Such a bump is necessary to better fit the B/C data [56], especially the HEAO data [81]. However, the AMS-02 data about B/C do not strongly favor a bump and hence the reacceleration for $E_{k} \lesssim 1 \mathrm{GeV} /$ nucleon [38]. The current data about $\mathrm{B} / \mathrm{C}$ by AMS-02 is not able to distinguish DR from DC model. Therefore to finally address the question that whether the reacceleration is favored one needs more precise measurement of the $\mathrm{B} / \mathrm{C}$ ratio down to sub-GeV. Similar conclusion has also been obtained in the study of the synchrotron radiation [82]. It was found that the DR model predicted higher radio emission than observed.

Finally, it is well known that the DM annihilation scenario of the positron excess is strongly constrained by the $\gamma$-ray observation. The exclusion limit derived from $\gamma$-ray observations of the dwarf galaxies by Fermi [83] and the Galactic center [84] are shown in Fig. 10. Similar to the conclusion in [9], the constraints from the Galactic center observation excludes all the DM scenarios. But this results suffer from large uncertainty of the small scale DM density profile. The constraints from the dwarf galaxies are much more solid. The DM annihilation to $\tau$ channel shows tension with the $\gamma$-ray observations. For $\mu$ channel, the current $\gamma$-ray data from the dwarf galaxies can still not be able to exclude the required parameter region to explain the lepton excess.

\section{CONCLUSIONS}

In this paper we give a global fitting to the AMS02 new results of the positron fraction, electron plus positron, electron and positron spectra to determine the primary electron spectrum as well as the extra $e^{+} e^{-}$ sources such as pulsars or DM. Two typical CR propagation models, DR and DC models, are discussed.

We find that in order to fit the data an additional break (hardening) at the primary electron spectrum at $\sim 60$ $\mathrm{GeV}$ is necessary. With such a primary electron spectrum, both the pulsar scenario and the DM scenario can give good fit to data, with $\chi^{2} /$ d.o.f. close to or smaller than 1 . The fittings are too worse to be acceptable without the additional hardening of the primary spectrum. The best case without the additional break is the DC scenario with pulsars as the extra source, which gives $\chi^{2} /$ d.o.f. $=374.2 / 264$ and corresponds to a $4.4 \sigma$ deviation from expectation.

In the two-break electron background case, both the pulsar and DM model can give good fittings to the AMS02 lepton data. If $\mathrm{DM}$ annihilate into $\mu$ final state the fitting value of its mass is about $0.4-1.5 \mathrm{TeV}$ and the annihilation cross section is about $(3-10) \times 10^{-24} \mathrm{~cm}^{3} \mathrm{~s}^{-1}$. For the $\tau$ final state the DM mass is about $1-7 \mathrm{TeV}$ and cross section is about $(2-20) \times 10^{-23} \mathrm{~cm}^{3} \mathrm{~s}^{-1}$. It is interesting to note that the DM scenario can reproduce the potential drop of the positron fraction data at $\sim 300$ $\mathrm{GeV}$ with the best fitting mass values.

We further find that to fit the lepton data the DC propagation model is more favorable than the $\mathrm{DR}$ model. This is because DR model will induce a bump at the local interstellar spectrum as a consequence of reacceleration. Such a bump is favored by the HEAO B/C data but is not favored by the lepton spectra. Therefore it is very important for the AMS-02 to give independent measurement of $\mathrm{B} / \mathrm{C}$ down to sub-GeV so as to determine the propagation model.

\section{Acknowledgments}

We thank Z.-H. Li, Z.-C. Tang, Z.-L. Weng and W.$\mathrm{W} . \mathrm{Xu}$ for helpful discussion. This work is supported by the NSFC under Grant Nos. 11475191, 11135009, and by the Strategic Priority Research Program "The Emergence of Cosmological Structures" of the Chinese Academy of Sciences, under Grant No. XDB09000000.

[1] O. Adriani et al. (PAMELA Collaboration), Nature 458, 607 (2009), 0810.4995.

[2] O. Adriani et al. (PAMELA Collaboration), 

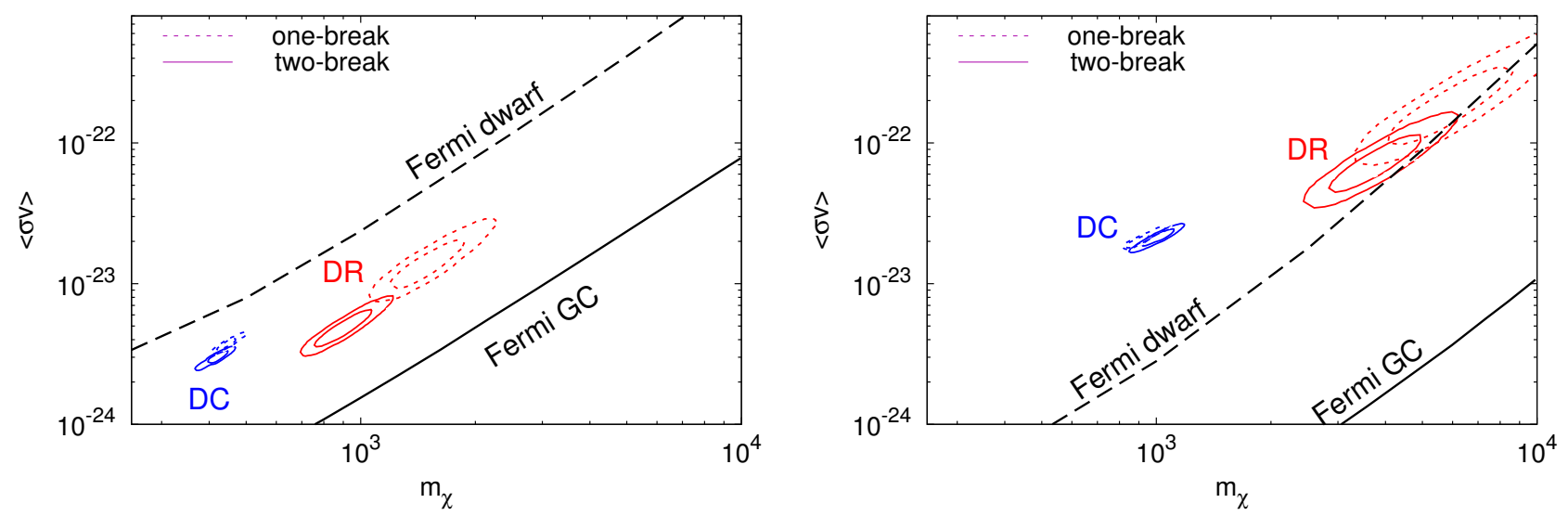

FIG. 10: $1 \sigma$ and $2 \sigma$ confidence regions in $m_{\chi}-\langle\sigma v\rangle$ plane, together with the exclusion lines from Fermi $\gamma$-ray observations $[83,84]$. The left panel is for $\mu$ channel, while the right panel is for $\tau$ channel. Blue contours are for DC scenario and red ones are for DR scenario. The dashed regions are for the case with only one break in the primary electron injection spectrum, while the solid regions are for those with an additional break.

Phys.Rev.Lett. 106, 201101 (2011), 1103.2880.

[3] A. A. Abdo et al. (Fermi LAT Collaboration), Phys.Rev.Lett. 102, 181101 (2009), 0905.0025.

[4] M. Ackermann et al. (Fermi LAT Collaboration), Phys.Rev. D82, 092004 (2010), 1008.3999.

[5] J. Chang, J. Adams, H. Ahn, G. Bashindzhagyan, M. Christl, et al., Nature 456, 362 (2008).

[6] F. Aharonian et al. (H.E.S.S. Collaboration), Phys.Rev.Lett. 101, 261104 (2008), 0811.3894.

[7] D. Borla Tridon, P. Colin, L. Cossio, M. Doro, and V. Scalzotto (MAGIC Collaboration) (2011), 1110.4008.

[8] M. Aguilar et al. (AMS Collaboration), Phys.Rev.Lett. 110, 141102 (2013).

[9] Q. Yuan, X.-J. Bi, G.-M. Chen, Y.-Q. Guo, S.-J. Lin, et al., Astropart.Phys. 60, 1 (2015), 1304.1482.

[10] L. Feng, R.-Z. Yang, H.-N. He, T.-K. Dong, Y.-Z. Fan, et al., Phys.Lett. B728, 250 (2014), 1303.0530.

[11] H.-B. Jin, Y.-L. Wu, and Y.-F. Zhou, JCAP 1311, 026 (2013), 1304.1997.

[12] M. Di Mauro, F. Donato, N. Fornengo, R. Lineros, and A. Vittino, JCAP 1404, 006 (2014), 1402.0321.

[13] S. Barwick et al. (HEAT Collaboration), Astrophys.J. 482, L191 (1997), astro-ph/9703192.

[14] M. Aguilar et al. (AMS-01 Collaboration), Phys.Lett. B646, 145 (2007), astro-ph/0703154.

[15] C. S. Shen, Astrophys. J. Lett. 162, L181 (1970).

[16] L. Zhang and K. S. Cheng, Astron. Astrophys. 368, 1063 (2001).

[17] H. Yuksel, M. D. Kistler, and T. Stanev, Phys.Rev.Lett. 103, 051101 (2009), 0810.2784.

[18] D. Hooper, P. Blasi, and P. D. Serpico, JCAP 0901, 025 (2009), 0810.1527.

[19] S. Profumo, Central Eur.J.Phys. 10, 1 (2011), 0812.4457.

[20] P. Blasi, Phys.Rev.Lett. 103, 051104 (2009), 0903.2794.

[21] H.-B. Hu, Q. Yuan, B. Wang, C. Fan, J.-L. Zhang, et al., Astrophys.J. 700, L170 (2009).

[22] Y. Fujita, K. Kohri, R. Yamazaki, K. Ioka, K. Kohri, et al., Phys.Rev. D80, 063003 (2009), 0903.5298.

[23] L. Bergstrom, T. Bringmann, and J. Edsjo, Phys.Rev. D78, 103520 (2008), 0808.3725.
[24] V. Barger, W. Y. Keung, D. Marfatia, and G. Shaughnessy, Phys.Lett. B672, 141 (2009), 0809.0162.

[25] M. Cirelli, M. Kadastik, M. Raidal, and A. Strumia, Nucl.Phys. B813, 1 (2009), 0809.2409.

[26] P.-f. Yin, Q. Yuan, J. Liu, J. Zhang, X.-j. Bi, et al., Phys.Rev. D79, 023512 (2009), 0811.0176.

[27] J. Zhang, X.-J. Bi, J. Liu, S.-M. Liu, P.-F. Yin, et al., Phys.Rev. D80, 023007 (2009), 0812.0522.

[28] L. Bergstrom, J. Edsjo, and G. Zaharijas, Phys.Rev.Lett. 103, 031103 (2009), 0905.0333.

[29] X.-G. He, Mod.Phys.Lett. A24, 2139 (2009), 0908.2908.

[30] Y.-Z. Fan, B. Zhang, and J. Chang, Int.J.Mod.Phys. D19, 2011 (2010), 1008.4646.

[31] P. D. Serpico, Astropart.Phys. 39-40, 2 (2012), 1108.4827.

[32] M. Cirelli, Pramana 79, 1021 (2012), 1202.1454.

[33] X.-J. Bi, P.-F. Yin, and Q. Yuan, Front.Phys.China 8, 794 (2013), 1409.4590.

[34] A. Lewis and S. Bridle, Phys.Rev. D66, 103511 (2002), astro-ph/0205436.

[35] J. Liu, Q. Yuan, X. Bi, H. Li, and X. Zhang, Phys.Rev. D81, 023516 (2010), 0906.3858.

[36] J. Liu, Q. Yuan, X.-J. Bi, H. Li, and X. Zhang, Phys.Rev. D85, 043507 (2012), 1106.3882.

[37] Q. Yuan and X.-J. Bi (2014), 1408.2424.

[38] AMS-02 collaboration, in International Cosmic Ray Conference (http://www.ams02.org/2013/07/newresults-from-ams-presented-at-icrc-2013/, 2013).

[39] L. Accardo et al. (AMS Collaboration), Phys.Rev.Lett. 113, 121101 (2014).

[40] M. Aguilar et al. (AMS Collaboration), Phys.Rev.Lett. 113, 121102 (2014).

[41] M. Aguilar et al. (AMS Collaboration), Phys.Rev.Lett. 113, 221102 (2014).

[42] V. S. Berezinskii, S. V. Bulanov, V. A. Dogiel, and V. S. Ptuskin, Astrophysics of cosmic rays (1990).

[43] E. S. Seo and V. S. Ptuskin, Astrophys. J. 431, 705 (1994).

[44] A. Strong and I. Moskalenko, Astrophys.J. 509, 212 (1998), astro-ph/9807150. 
[45] I. Moskalenko and A. Strong, Astrophys.J. 493, 694 (1998), astro-ph/9710124.

[46] R. A. Mewaldt, J. R. Jokipii, M. A. Lee, E. Möbius, and T. H. Zurbuchen, eds., Acceleration and Transport of Energetic Particles Observed in the Heliosphere., vol. 528 of American Institute of Physics Conference Series (2000).

[47] N. E. Yanasak, M. E. Wiedenbeck, R. A. Mewaldt, A. J. Davis, A. C. Cummings, J. S. George, R. A. Leske, E. C. Stone, E. R. Christian, T. T. von Rosenvinge, et al., Astrophys. J. 563, 768 (2001).

[48] F. A. Hagen, A. J. Fisher, and J. F. Ormes, Astrophys. J. 212, 262 (1977), URL http: //adsabs .harvard. edu/abs/1977ApJ . . 212. .262H.

[49] A. Buffington, C. D. Orth, and T. S. Mast, Astrophys. J. 226, 355 (1978), URL http://adsabs . harvard.edu/abs/1978ApJ . . 226 . .355B.

[50] W. R. Webber and J. Kish, International Cosmic Ray Conference 1, 389 (1979), URL http: //adsabs . harvard. edu/abs/1979ICRC . . . 1. . 389W.

[51] M. Garcia-Munoz, J. A. Simpson, and J. P. Wefel, International Cosmic Ray Conference 2, 72 (1981).

[52] J. A. Simpson and M. Garcia-Munoz, Space Sci. Rev. 46, 205 (1988).

[53] T. Hams, L. M. Barbier, M. Bremerich, E. R. Christian, G. A. de Nolfo, S. Geier, H. Göbel, S. K. Gupta, M. Hof, W. Menn, et al., Astrophys. J. 611, 892 (2004).

[54] J. J. Connell, Astrophys. J. Lett. 501, L59 (1998).

[55] A. Lukasiak, International Cosmic Ray Conference 3, 41 (1999).

[56] I. V. Moskalenko, A. W. Strong, J. F. Ormes, and M. S. Potgieter, Astrophys.J. 565, 280 (2002), astro$\mathrm{ph} / 0106567$.

[57] L. Gleeson and W. Axford, Astrophys.J. 154, 1011 (1968).

[58] A. W. Strong, I. V. Moskalenko, and V. S. Ptuskin, Ann.Rev.Nucl.Part.Sci. 57, 285 (2007), astroph/0701517.

[59] M. Ackermann, M. Ajello, W. B. Atwood, L. Baldini, J. Ballet, G. Barbiellini, D. Bastieri, K. Bechtol, R. Bellazzini, B. Berenji, et al., Astrophys. J. 750, 3 (2012).

[60] R. Trotta, G. Johannesson, I. Moskalenko, T. Porter, R. R. de Austri, et al., Astrophys.J. 729, 106 (2011), 1011.0037.

[61] T. Kamae, N. Karlsson, T. Mizuno, T. Abe, and T. Koi, Astrophys.J. 647, 692 (2006), astro-ph/0605581.

[62] T. Delahaye, F. Donato, N. Fornengo, J. Lavalle,
R. Lineros, et al., Astron.Astrophys. 501, 821 (2009), 0809.5268 .

[63] M. Mori, Astropart.Phys. 31, 341 (2009), 0903.3260.

[64] Q. Yuan and X.-J. Bi, Phys.Lett. B727, 1 (2013), 1304.2687.

[65] I. Cholis and D. Hooper, Phys.Rev. D88, 023013 (2013), 1304.1840.

[66] D. R. Lorimer, in Young Neutron Stars and Their Environments, edited by F. Camilo \& B. M. Gaensler (2004), vol. 218 of IAU Symposium, p. 105.

[67] O. Adriani et al. (PAMELA Collaboration), Phys.Rev.Lett. 105, 121101 (2010), 1007.0821.

[68] M. Cirelli, G. Corcella, A. Hektor, G. Hutsi, M. Kadastik, et al., JCAP 1103, 051 (2011), 1012.4515.

[69] P. Ciafaloni, D. Comelli, A. Riotto, F. Sala, A. Strumia, et al., JCAP 1103, 019 (2011), 1009.0224.

[70] J. F. Navarro, C. S. Frenk, and S. D. White, Astrophys.J. 490, 493 (1997), astro-ph/9611107.

[71] S. P. Reynolds, Astrophys. J. 327, 853 (1988).

[72] D. Thompson, Z. Arzoumanian, D. Bertsch, K. Brazier, J. Chiang, et al., Astrophys.J. 436, 229 (1994).

[73] J. Fierro, Z. Arzoumanian, M. Bailes, J. Bell, D. Bertsch, et al., Astrophys.J. 447, 807 (1995).

[74] A. D. Panov, J. Adams, H. Ahn, G. Bashindzhagyan, K. Batkov, et al., Bull.Russ.Acad.Sci.Phys. 71, 494 (2007), astro-ph/0612377.

[75] H. Ahn, P. Allison, M. Bagliesi, J. Beatty, G. Bigongiari, et al., Astrophys.J. 714, L89 (2010), 1004.1123.

[76] O. Adriani et al. (PAMELA Collaboration), Science 332, 69 (2011), 1103.4055.

[77] Q. Yuan, B. Zhang, and X.-J. Bi, Phys.Rev. D84, 043002 (2011), 1104.3357.

[78] S. Zhang (the HERD collaboration) (2014), 1407.4866.

[79] P.-F. Yin, Z.-H. Yu, Q. Yuan, and X.-J. Bi, Phys.Rev. D88, 023001 (2013), 1304.4128.

[80] T. Linden and S. Profumo, Astrophys.J. 772, 18 (2013), 1304.1791.

[81] J. J. Engelmann, P. Ferrando, A. Soutoul, P. Goret, and E. Juliusson, Astron. Astrophys. 233, 96 (1990).

[82] A. Strong, E. Orlando, and T. Jaffe, Astron.Astrophys. 534, A54 (2011), 1108.4822.

[83] M. Ackermann et al. (Fermi-LAT Collaboration), Phys.Rev. D89, 042001 (2014), 1310.0828.

[84] X. Huang, Q. Yuan, P.-F. Yin, X.-J. Bi, and X. Chen, JCAP 1211, 048 (2012), 1208.0267. 